\title{
Length-weight relationships for the fifty littoral and coastal marine fish species from the Eastern Adriatic sea
}

\author{
Alen SOLDO \\ Department of Marine Studies, University of Split, Croatia \\ Corresponding autor,e-mail: soldo@unist.hr
}

The length-weight relationship parameters are reported for the fifty littoral and coastal marine fish species from the Eastern Adriatic. Captures were made between the years 2004 and 2017 during sport fishing competitions. The parameters $a$ and $b$ of the equation $W=a L^{b}$ were estimated. The $b$ values ranged from 2.1972 for Spicara smaris (Linnaeus, 1758) to 3.7044 for Salaria pavo (Risso, 1810). The L-W relation for Scyliorhinus stellaris (Linnaeus, 1758), Gobius bucchichi Steindachner 1870, Gobius geniporus Valenciennes 1837 and Lipophrys trigloides (Valenciennes, 1836) are not available in FishBase and presents new data for these species. For some other species, such as Labrus mixtus Linnaeus 1758, there are only two relationships, but none from the Mediterranean, for Gobius paganellus Linnaeus 1758 only four relationships and none from Central and Eastern Mediterranean, while for Labrus merula Linnaeus 1758, Symphodus mediterraneus (Linnaeus, 1758), Symphodus roissali (Risso, 1810), Gobius cobitis Pallas 1814, Gobius cruentatus Gmelin 1789, Gobius niger Linnaeus 1758, Parablennius gatorugine (Linnaeus, 1758), Parablennius sanguinolentus (Pallas, 1814), Salaria pavo (Risso, 1810), and Bothus podas (Delaroche, 1809) very low number of relationships are presented and none from the Adriatic Sea.

Key words: length-weight relationship, parameters, fish, littoral and coastal, Adriatic

\section{INTRODUCTION}

The length-weight relationship (LWR) is useful in fishery assessments (ÇALIK \&, ERDOĞAN SAĞLAM, 2017) for predicting weights from the more easily measured lengths (RICKER, 1975), yield assessment (GARCIA et al., 1998) and when calculating biomass (MARTIN-SMITH, 1996). During various sampling programs, it is usually easier to measure only length (e.g., because of the bobbing motion of the boat), or weight cannot be measured at all (e.g., underwater visual censuses). The LWR of a particular species allows the inter-conversion of these parameters. The relationship between the length (L) and weight (W) of a fish is usually expressed by the equation $\mathrm{W}=a \mathrm{~L}^{b}$. The exponent $b$ provides information on growth (MOREY et al., 2003); being isometric when $b=3$ and allometric when this is not the case (positive if $b>3$, negative if $b<3$ ). The present study provides information on the length-weight relationships of fifty littoral and coastal marine fish species from the littoral and coastal waters of the Eastern Adriatic Sea.

\section{MATERIAL AND METHODS}

Data were collected between 2004 and 2017 from the Eastern Adriatic sea during the study of sport fishing competitions, using various fishing 
gears, from the different hook lines to spear fishing. Competitions are held from the shore, and boats, while spear fishermen were fishing near shore or further offshore on underwater reefs. Thus, most of the fishes have been caught up to $40 \mathrm{~m}$ of depth. Due to the common period of the sport fishing competitions all fishes were collected from May until the end of October. Data on total length (TL, cm) to the nearest millimeter and total weight $(\mathrm{TW}, \mathrm{g})$ to the nearest gram were recorded for each fish. The length-weight relationship was calculated using the expression: $\mathrm{W}=a \mathrm{~L}^{b}$, where the $\mathrm{W}$ is the weight $(\mathrm{g}), \mathrm{L}$ the length (cm), $a$ the intercept of the regression and $b$ is the regression coefficient (HAYES et al., 1995). Parameters $a$ and $b$ of the length-weight relationship were estimated by linear regression analysis based on logarithms: $\log (\mathrm{W})=\log (a)$ $+b \log (\mathrm{L})$.

\section{RESULTS AND DISCUSSION}

A total of 16373 fish specimens representing 20 families and 50 different fish species were analyzed. For each species, the sample size, the minimum and maximum total length, the minimum and maximum weight measured, the LWR parameters $a$ and $b$, and the coefficient of determination $\mathrm{r}^{2}$ are presented in Table 1 . The L-W relationship for Scyliorhinus stellaris (Linnaeus, 1758), Gobius bucchichi Steindachner 1870, Gobius geniporus Valenciennes 1837 and Lipophrys trigloides (Valenciennes, 1836) are not available in FishBase (FROESE \& PAULY, 2019). For some other species, such as Labrus mixtus Linnaeus 1758, there are only 2 relationships, none from the Mediterranean, for Gobius paganellus Linnaeus 1758 only 4 relationships and none from Central and Eastern Mediterranean, while for Labrus merula Linnaeus 1758, Symphodus mediterraneus (Linnaeus, 1758), Symphodus roissali (Risso, 1810), Gobius cobitis Pallas 1814, Gobius cruentatus Gmelin 1789, Gobius niger Linnaeus 1758, Parablennius gatorugine (Linnaeus, 1758), Parablennius sanguinolentus (Pallas, 1814), Salaria pavo (Risso, 1810), Trachinus radiatus Cuvier 1829 and Bothus podas (Delaroche, 1809) very low num- ber of relationships are presented and none from the Adriatic Sea, except for T. radiates (FROESE \& PAULY, 2019). Comparing maximum lengths of fishes indicated in FishBase (FROESE \& PAULY, 2019) with those obtained during this study, the new maximum lengths can be reported for a range of gobies, particularly G. cobitis (30.0 $\mathrm{cm})$, G. bucchichi $(12.1 \mathrm{~cm})$, G. geniporus (17.8 $\mathrm{cm})$, G. paganellus $(16.0 \mathrm{~cm})$ and for a blenny $S$. pavo $(13.4 \mathrm{~cm})$. The sample size ranged from 61 specimens for Muraena helena Linnaeus 1758 to 1472 for Coris julis (Linnaeus, 1758). The $\mathrm{r}^{2}$ values ranged from 0.5644 for Spicara smaris (Linnaeus, 1758) to 0.9980 for Chelidonichthys lastoviza (Bonnaterre, 1788), 0,9953 for $T$. radiatus and 0.9917 for Sarpa salpa (Linnaeus, 1758). Twenty seven species, out of 50 , showed $\mathrm{r}^{2}$ values higher than 0.95 , while only four of them had $\mathrm{r}^{2}$ values less than 0.75 . The $b$ values ranged from 2.1972 for $S$. smaris to 3.7044 for $S$. pavo. Three species had $b$ values less than 2.5 while only one had higher than 3.5. The mean value of $b$ was 2.975 , while the median was 2.9804. $50 \%$ of the $b$ values ranged between 2.8473 and 3.1434 .

BAGENAL \& TESCH (1978) reported that the parameters of weight-length relationships, particularly $a$, may vary daily, seasonally, and/or between habitats, unlike the parameter $b$, which does not vary significantly throughout the year. However, the comparison of $b$ values for the same species appearing in different studies in the Mediterranean Sea suggested inter-regional differences (MOREY et al., 2003). In order to test differences on a regional level, $b$ values for similar habitat and species obtained previously from the Adriatic (DULČIĆ \& KRALJEVIĆ, 1996) were compared to results of this study (Table 2). Comparison showed that for most of the species $b$ values were indeed similar, but for Mullus surmuletus Linnaeus 1758 (3.512 vs 3,0901), Pagellus acarne (Risso, 1827) (3,499 vs 2,746) and Scorpaena scrofa Linnaeus 1758 (3,298 vs 2,8162 ) were significantly different. Therefore, it can be concluded that even $b$ values, although less than $a$ values, can vary due to a combination of different reasons, such as period of the year, spatial variation, influence of water qual- 
ity, food availability, population differences and the ontogenetic stage of the individuals studied (SPARRE et al., 1989, MOMMSEN, 1998, DULČIĆ \& GLAMUZINA, 2006) or differences in sampling (the number of specimens and length ranges).
Thus, when LWR parameters are used for some species it would be appropriate, for obtaining more precise weight estimations, to use local/regional values calculated within suitable length ranges.

Table 1. Length - weight relationship parameters for the fifty species from the Eastern Adriatic

\begin{tabular}{|c|c|c|c|c|c|c|}
\hline Family/Species & $\mathrm{N}$ & $\begin{array}{l}\text { Weight range } \\
(\mathrm{g})\end{array}$ & $\begin{array}{l}\text { Length range } \\
(\mathrm{cm})\end{array}$ & $\mathrm{a}$ & $\mathrm{b}$ & $r^{2}$ \\
\hline \multicolumn{7}{|l|}{ Scyliorhinidae } \\
\hline Scyliorhinus stellaris & 86 & $998-3820$ & $58,1-96,2$ & 0,0705 & 2,3769 & 0,8161 \\
\hline \multicolumn{7}{|l|}{ Sparidae } \\
\hline Boops boops & 1144 & $12-236$ & $11,0-27,5$ & 0,0147 & 2,8608 & 0,9515 \\
\hline Dentex dentex & 92 & $442-6695$ & $32,7-82,1$ & 0,0095 & 3,0855 & 0,9884 \\
\hline Diplodus annularis & 1325 & $8-195$ & $8,3-22,5$ & 0,017 & 2,9796 & 0,9511 \\
\hline Diplodus puntazzo & 101 & $61-795$ & $15,6-39,0$ & 0,0243 & 2,8477 & 0,9772 \\
\hline Diplodus sargus & 415 & $58-1525$ & $15,3-42,0$ & 0,0251 & 2,9003 & 0,9322 \\
\hline Diplodus vulgaris & 740 & $8-570$ & $8,5-32,0$ & 0,0118 & 3,0926 & 0,9789 \\
\hline Lithognathus mormyrus & 74 & $41-488$ & $15,0-32,7$ & 0,008 & 3,1393 & 0,9849 \\
\hline Pagellus erythrinus & 1235 & $12-744$ & $10,0-39,2$ & 0,0163 & 2,8942 & 0,9786 \\
\hline Pagellus acarne & 288 & $12-110$ & $10,0-21,1$ & 0,0253 & 2,746 & 0,8847 \\
\hline Sarpa salpa & 171 & $16-885$ & $10,7-39,0$ & 0,015 & 2,9636 & 0,9917 \\
\hline Sparus aurata & 202 & $124-1460$ & $21,0-49,1$ & 0,0106 & 3,064 & 0,9542 \\
\hline \multicolumn{7}{|l|}{ Labridae } \\
\hline Coris julis & 1472 & $4-116$ & $8,5-23,0$ & 0,0035 & 3,3611 & 0,967 \\
\hline Labrus merula & 299 & $59-1102$ & $16,6-42,0$ & 0,0183 & 2,947 & 0,9457 \\
\hline Labrus mixtus & 63 & $60-536$ & $18,1-34,6$ & 0,0042 & 3,3251 & 0,9786 \\
\hline Symphodus mediterraneus & 301 & $10-64$ & $8,0-16,1$ & 0,0247 & 2,7591 & 0,8226 \\
\hline Symphodus roissali & 93 & $10-41$ & $8,4-13,4$ & 0,0109 & 3,1801 & 0,867 \\
\hline Symphodus tinca & 876 & $14-565$ & $9,6-34,8$ & 0,0212 & 2,8351 & 0,9636 \\
\hline \multicolumn{7}{|l|}{ Gobiidae } \\
\hline Gobius bucchichi & 62 & $8-18$ & $9,1-12,1$ & 0,0071 & 3,1548 & 0,7205 \\
\hline Gobius cobitis & 141 & $12-332$ & $10,3-30,0$ & 0,0134 & 2,9844 & 0,9609 \\
\hline Gobius cruentatus & 504 & $8-78$ & $9,6-17,8$ & 0,0078 & 3,1435 & 0,9002 \\
\hline Gobius geniporus & 472 & $4-58$ & $7,2-17,2$ & 0,0546 & 2,36 & 0,7784 \\
\hline Gobius niger & 91 & $5-31$ & $7,8-14,0$ & 0,0092 & 3,0831 & 0,8622 \\
\hline Gobius paganellus & 277 & $8-38$ & $9,8-16,0$ & 0,028 & 2,6675 & 0,7496 \\
\hline \multicolumn{7}{|l|}{ Serranidae } \\
\hline Serranus cabrilla & 457 & $12-226$ & $10,8-26,2$ & 0,0123 & 2,9636 & 0,9561 \\
\hline Serranus hepatus & 169 & $3-32$ & $5,3-12,0$ & 0,0364 & 2,6475 & 0,8977 \\
\hline Serranus scriba & 923 & $10-154$ & $9,8-22,1$ & 0,0092 & 3,1433 & 0,9621 \\
\hline Centracanthidae & & & & & & \\
\hline
\end{tabular}




\begin{tabular}{|c|c|c|c|c|c|c|}
\hline Spicara flexuosa & 504 & $17-92$ & $12,0-19,8$ & 0,0093 & 3,0367 & 0,8639 \\
\hline Spicara maena & 182 & $32-340$ & $14,2-27,1$ & 0,0052 & 3,2846 & 0,9526 \\
\hline Spicara smaris & 179 & $22-52$ & $12,3-19,2$ & 0,0716 & 2,1972 & 0,5644 \\
\hline \multicolumn{7}{|l|}{ Scorpaenidae } \\
\hline Scorpaena porcus & 173 & $21-770$ & $10,5-32,7$ & 0,0099 & 3,2133 & 0,9841 \\
\hline Scorpaena scrofa & 225 & $20-2060$ & $10,6-50,2$ & 0,034 & 2,8162 & 0,9542 \\
\hline \multicolumn{7}{|l|}{ Carangidae } \\
\hline Trachurus trachurus & 377 & $20-564$ & $13,4-44,5$ & 0,0115 & 2,8637 & 0,9746 \\
\hline \multicolumn{7}{|l|}{ Blenniidae } \\
\hline Lipophrys trigloides & 77 & $9-26$ & $8,9-13,0$ & 0,0341 & 2,5688 & 0,7557 \\
\hline Parablennius gatorugine & 155 & $6-198$ & $7,4-22,0$ & 0,0064 & 3,2741 & 0,9667 \\
\hline Parablennius sanguinolentus & 316 & $9-89$ & $9,7-19,4$ & 0,0051 & 3,343 & 0,9261 \\
\hline Salaria pavo & 83 & $4-16$ & $8,3-13,4$ & 0,0015 & 3,7044 & 0,9215 \\
\hline \multicolumn{7}{|l|}{ Mugilidae } \\
\hline Chelon auratus & 83 & $205-875$ & $27,6-47,5$ & 0,0158 & 2,8472 & 0,9029 \\
\hline Chelon ramada & 65 & $278-1311$ & $35,5-56,8$ & 0,0243 & 2,7089 & 0,9707 \\
\hline \multicolumn{7}{|l|}{ Triglidae } \\
\hline Chelidonichthys lastoviza & 64 & $40-214$ & $15,8-27,7$ & 0,0133 & 2,9109 & 0,998 \\
\hline \multicolumn{7}{|l|}{ Trachinidae } \\
\hline Trachinus draco & 447 & $7-192$ & $10,4-32,2$ & 0,0082 & 2,9063 & 0,9784 \\
\hline Trachinus radiatus & 85 & $24-399$ & $13,8-33,5$ & 0,0085 & 3,0285 & 0,9953 \\
\hline \multicolumn{7}{|l|}{ Congridae } \\
\hline Conger conger & 391 & $735-11775$ & $70,9-165,4$ & 0,0006 & 3,2754 & 0,9057 \\
\hline \multicolumn{7}{|l|}{ Phycidae } \\
\hline Phycis phycis & 127 & $210-2720$ & $26,6-58,3$ & 0,0048 & 3,2499 & 0,9594 \\
\hline \multicolumn{7}{|l|}{ Mullidae } \\
\hline Mullus surmuletus & 157 & $14-330$ & $9,9-30,3$ & 0,0099 & 3,0901 & 0,9797 \\
\hline \multicolumn{7}{|l|}{ Sciaenidae } \\
\hline Sciaena umbra & 118 & $328-1995$ & $29,0-52,2$ & 0,0095 & 3,1053 & 0,9664 \\
\hline \multicolumn{7}{|l|}{ Pomacenthridae } \\
\hline Chromis chromis & 235 & $11-48$ & $9,2-13,2$ & 0,0181 & 2,979 & 0,6425 \\
\hline \multicolumn{7}{|l|}{ Moronidae } \\
\hline Dicentrarchus labrax & 119 & $230-2963$ & $27,8-64,1$ & 0,0104 & 2,9812 & 0,9095 \\
\hline \multicolumn{7}{|l|}{ Muraenidae } \\
\hline Muraena helena & 61 & $1115-4310$ & $79,0-120,2$ & 0,0116 & 2,6477 & 0,8212 \\
\hline \multicolumn{7}{|l|}{ Bothidae } \\
\hline Bothus podas & 77 & $16-58$ & $12,6-18,1$ & 0,0058 & 3,2125 & 0,8998 \\
\hline
\end{tabular}

$n$-sample size, weight range, total length range, $a$ and $b$ parameters, $r 2$ - coefficient of determination 
Table 2. Number of specimens (n), total length ranges (TL range) and b values for species compared between previous study from the Adriatic (DULČIĆ \& KRALJEVIĆ, 1996) and present study

\begin{tabular}{|c|c|c|c|c|c|c|}
\hline \multirow[t]{2}{*}{ species } & \multicolumn{3}{|c|}{ DULČIĆ \& KRALJEVIĆ, 1996} & \multicolumn{3}{|c|}{ present study } \\
\hline & $\mathrm{n}$ & TL range $(\mathrm{cm})$ & $\mathrm{b}$ & $\mathrm{n}$ & TL range $(\mathrm{cm})$ & $\mathrm{b}$ \\
\hline Chromis chromis & 1230 & $5,9-13,9$ & 3,102 & 235 & $9,2-13,2$ & 2,979 \\
\hline Coris julis & 94 & $4,3-15,3$ & 3,238 & 1472 & $8,5-23,0$ & 3,3611 \\
\hline Dentex dentex & 250 & $18,3-85,9$ & 3,172 & 92 & $32,7-82,1$ & 3,0855 \\
\hline Dicentrarchus labrax & 417 & $24,5-88,0$ & 3,146 & 119 & $27,8-64,1$ & 2,9812 \\
\hline Diplodus annularis & 100 & $8,9-23,5$ & 2,928 & 1325 & $8,3-22,5$ & 2,9796 \\
\hline Diplodus puntazzo & 289 & $5,9-45,0$ & 2,951 & 101 & $15,6-39,0$ & 2,8477 \\
\hline Diplodus sargus & 214 & $14,3-39,9$ & 3,038 & 415 & $15,3-42,0$ & 2,9003 \\
\hline Diplodus vulgaris & 601 & $14,3-39,9$ & 3,028 & 740 & $8,5-32,0$ & 3,0926 \\
\hline Mullus surmuletus & 127 & $15,4-30,9$ & 3,512 & 157 & $9,9-30,3$ & 3,0901 \\
\hline Pagellus acarne & 74 & $10,0-23,7$ & 3,499 & 288 & $10,0-21,1$ & 2,746 \\
\hline Pagellus erythrinus & 193 & $10,9-46,0$ & 2,944 & 1235 & $10,0-39,2$ & 2,8942 \\
\hline Phycis phycis & 58 & $26,2-56,4$ & 3,502 & 127 & $26,6-58,3$ & 3,2499 \\
\hline Sciaena umbra & 26 & $18,1-41,2$ & 3,048 & 118 & $29,0-52,2$ & 3,1053 \\
\hline Scorpaena porcus & 351 & $9,7-26,6$ & 3,243 & 173 & $10,5-32,7$ & 3,2133 \\
\hline Scorpaena scrofa & 125 & $19,7-53,6$ & 3,298 & 225 & $10,6-50,2$ & 2,8162 \\
\hline Spicara maena & 220 & $14,2-27,5$ & 3,037 & 182 & $14,2-27,1$ & 3,2846 \\
\hline Symphodus tinca & 100 & $12,7-30,2$ & 2,726 & 876 & $9,6-34,8$ & 2,8351 \\
\hline Trachinus draco & 22 & $9,2-26,8$ & 2,934 & 447 & $10,4-32,2$ & 2,9063 \\
\hline Trigloporus lastoviza & 52 & $7,4-35,0$ & 3,003 & 64 & $15,8-27,7$ & 2,9109 \\
\hline
\end{tabular}

\section{REFERENCES}

BAGENAL, T.B. \& P.W. TESCH, 1978. Age and growth. In: Methods for Assessment of Fish in Fresh Waters. $3^{\text {rd }}$ Edition (Edittor T. Benegal), IBP Handbook No. 3. Blackwell Scientific Publications, Oxford, pp. 101-136.

ÇALIK, S. \& N. ERDOĞAN SAĞLAM. 2017. Lengthweight relationships of demersal fish species caught by bottom trawl from Eastern Black Sea (Turkey). Cahiers de Biologie Marine, 58: 485 - 490 DOI: 10.21411/ CBM.A.AA0D91E6

DULČIĆ, J. \& B. GLAMUZINA. 2006. Length-weight relationships for selected fish species from three eastern Adriatic estuarine systems (Croatia). J. Appl. Ichthyol. 22: 254-256 DULČIĆ, J. \& M. KRALJEVIĆ 1996. Weight-length relationships for fish species in the eastern Adriatic (Croatian waters). Fisheries Research, 28: 243-251. DOI: 10.1016/01657836(96)00513-9

FROESE, R. \& D. PAULY. (Editors) 2019. FishBase. World Wide Web electronic publication. Www.fishbase.org, (10/2019)

GARCIA, C.B., DUARTE, J.O., SANDOVAL, N., VON SCHILLER, D., MELO, G. \& P. NAVAJAS 1998. Length-weight relationships of demersal fishes from the Gulf of Salamanca, Colombia, Naga. ICLARM Quart., 21 (3): 30-32.

HAYES, D.B., BRODZIAK, J.K.T. \& J.B. O'GORMAN 1995. Efficiency and bias of estimators and sampling designs for determining lengthweight relationships of fish. Canadian Jour- 
nal of Fisheries and Aquatic Science, 52: 84-92. DOI: 10.1139/f95-008

MARTIN-SMITH, K.H. 1996. Length/weight relationships of fishes in a diverse tropical freshwater community, Sabah, Malaysia. Journal of Fish Biology, 49: 731-734. DOI: 10.1111/ j.1095-8649.1996.tb00069.x

MOMMSEN, T.P. 1998. Growth and metabolism. In: The Physiology of Fishes (Evans D.H. ed), pp. 65-97. CRC Press, New York.

MOREY, G., MORANTA, J., MASSUTI, E., GRAU, A., LINDE, M., RIERA, F. \& B. MORALES-NIN. 2003.
Weight-length relationships of littoral to lower slope fishes from the western Mediterranean. Fisheries Research, 62: 89-96. DOI: 10.1016/S0165-7836(02)00250-3

RICKER, W.E. 1975. Computation and interpretation of biological statistics of fish populations. Bulletin of the Fisheries Research Board of Canada, 191: $382 \mathrm{p}$.

SPARRE, P., URSIN, E. \& S.C. VENEMA. 1989. Introduction to tropical fish stock assessment. Part I. Manual. FAO Fisheries Technical Paper No. 306.337 pp.

Received: 18 November 2019

Accepted: 15 October 2020

\title{
Dužinsko-maseni odnos pedeset litoralnih i obalnih riba u istočnom Jadranu
}

\section{Alen SOLDO}

\author{
Kontakte-pošta: soldo@unist.hr
}

\section{SAŽETAK}

Parametri dužinsko-masenog odnosa su prijavljeni za 50 litoralnih i obalnih rba iz istočnog Jadrana. Svi primjerci su ulovljeni u periodu 2004-2017 za vrijeme natjecanja u sportskom ribolovu.

Parameteri $a$ i $b$ od jednadžbe $\mathrm{W}=\mathrm{aL}^{\mathrm{b}}$ su prezentirani. Vrijednosti $b$ parametra su varirale od 2.1972 za Spicara smaris (Linnaeus, 1758) do 3.7044 za Salaria pavo (Risso, 1810). Dužinskomaseni odnosi za Scyliorhinus stellaris (Linnaeus, 1758), Gobius bucchichi Steindachner 1870, Gobius geniporus Valenciennes 1837 i Lipophrys trigloides (Valenciennes, 1836) dosad nisu bili dostupni u FishBase.

Za neke druge vrste, primjerice Labrus mixtus Linnaeus 1758, postoje samo dva prijavljena odnosa, pri čemu niti jedan nije iz Mediterana, za Gobius paganellus Linnaeus 1758 postoje samo 4 odnosa i nijedan iz središnjeg i istočnog Mediterana, dok za Labrus merula Linnaeus 1758, Symphodus mediterraneus (Linnaeus, 1758), Symphodus roissali (Risso, 1810), Gobius cobitis Pallas 1814, Gobius cruentatus Gmelin 1789, Gobius niger Linnaeus 1758, Parablennius gatorugine (Linnaeus, 1758), Parablennius sanguinolentus (Pallas, 1814), Salaria pavo (Risso, 1810) i Bothus podas (Delaroche, 1809) postoji veoma mali broj dužinsko-masenih odnosa, pri čemu niti jedan nije prijavljen za Jadransko more, osim za T. radiates.

Ključne riječi: dužinsko-maseni odnos, parametri, riba, primorje i priobalje, Jadran 